\title{
Intravital Microfluidic Windows for Delivery of Chemicals, Drugs and Probes
}

\author{
P. Myneni ${ }^{1}$, A. Terekhov ${ }^{2}$, G. Wright ${ }^{1}$, W. Hofmeister ${ }^{2}$, C. Janetopoulos ${ }^{1,3}$
}

1 Department of Biological Sciences, Vanderbilt University, Nashville, Tennessee 37240, USA.

2 Center for Laser Applications, University of Tennessee Space Institute,Tullahoma, Tennessee 37388, USA.

3 Department of Cell \& Developmental Biology, Vanderbilt University School of Medicine Nashville, Tennessee 37240, USA.

Intravital imaging, the microscopic visualization of cellular events in living animals, is becoming a powerful tool for the study of many disease processes. The development of the mammary imaging window has been shown to be advantageous for high resolution imaging for the study of cell movements within an organism and can be used to allow the precise observation of cells and tumor angiogenesis for many days [1,2] [3]. A major disadvantage of intravital imaging, however, is that the imaging windows are typically sewn into the skin of the animal, and therefore, the imaging area is not amendable to chemical or drug treatments. The addition of local agents by syringe needle is problematic as injection can elicit unwanted wound responses that complicate elucidation of cellular-response pathways to both secreted factors and pharmacological agents. Systemic treatments can be harmful to the animal and reagents may not make their way to the area under observation. We have designed microfluidic glass windows that will alleviate these complications and provide real time spatial and temporal addition of chemical treatments to cells and tissues.

Our system allows the careful titration of reagents to various tissues during imaging. While we can make micron-sized ports for setting up gradients, we can also make larger openings for the addition of various labeled cell types to watch their infiltration into a various organ or tissue. Such a system, for instance, would also allow the addition of controlled amounts of pathogens to visualize a site of infection and the immune response.

With glass, it is possible to etch channels and ports down to a few hundred nanometers [4]. They are also durable, optically superior, easy to unclog and fill, and are reusable. Channels were fabricated using a laser ablation system which consists of a diode-pumped frequency doubled Neodymium-doped Yttrium Orthovanadate (Nd:YVO4) laser pump (Verdi), a Ti:Sapphire laser oscillator (Tsunami) and a regenerative amplifier (RegA9000). The substrate position was controlled by an Aerotech ANT95 nanopositioning system and is capable of carefully coordinating the alignment and positioning of the design features through software-controlled stages with nanometer precision.

We have previously shown that we can make several chemotaxis devices in glass coverslips [5]. Here, we demonstrate the usefulness of intravital microfluidic windows by staining subcellular organelles in tissues, obtaining cellular responses by adding ligands, and have also set up chemical gradients, all in a living animal. We have imaged tissues and cells with epifluorescence and 2 photon microscopy. In addition to adding reagents, we can also potentially collect cells and effluent for histological and proteomic analysis during and after chemical treatments. 
[1] Le Devedec, S.E., Yan, K., de Bont, H., Ghotra, V., Truong, H., Danen, E.H., Verbeek, F., and van de Water, B. Systems microscopy approaches to understand cancer cell migration and metastasis. Cell Mol Life Sci 67, 3219-3240.

[2] Kedrin, D., Gligorijevic, B., Wyckoff, J., Verkhusha, V.V., Condeelis, J., Segall, J.E., and van Rheenen, J. (2008). Intravital imaging of metastatic behavior through a mammary imaging window. Nat Methods 5, 1019-1021.

[3] Hak, S., Reitan, N.K., Haraldseth, O., and de Lange Davies, C. Intravital microscopy in window chambers: a unique tool to study tumor angiogenesis and delivery of nanoparticles. Angiogenesis 13, 113-130.

[4] Costa, L.T., Terekhov, A., Rajput, D., Hofmeister, W., Jowhar, D., Wright, G., and Janetopoulos, C. (2011). Femtosecond laser machined microfluidic devices for imaging of cells during chemotaxis. Journal of Laser Applications 23, 042001-042006.

[5] Wright, G.A., Costa, L., Terekhov, A., Jowhar, D., Hofmeister, W., and Janetopoulos, C. (2012). On-chip open microfluidic devices for chemotaxis studies. Microsc Microanal 18, 816-828.

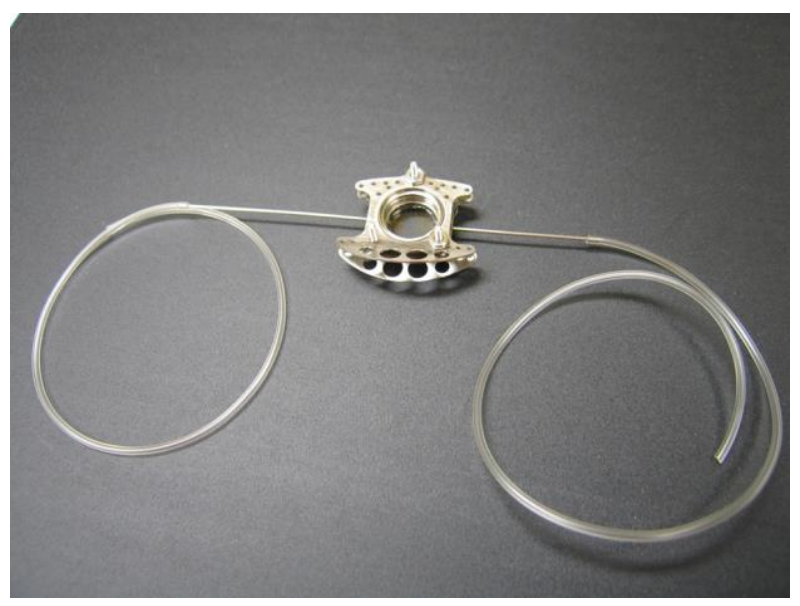

Figure 1. Glass viewport is in a stainless ring with tubes attached so there is no micro-plumbing on assembly. A microfluidic channel runs through the center of the window with ports facing the animal. Four screws hold view/fluidport assembly to the frame. This device has been successfully tested on mice. 